حساب القيمة المتوقعة لشحنة الاككترون للانظمة الذرية ذات الاككترونين في فضاء

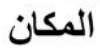

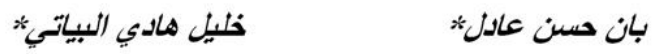

تاريخ قبول النشر 28/ 2 / 2010

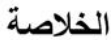

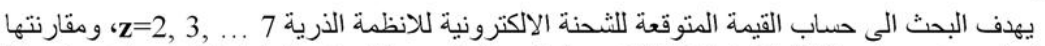

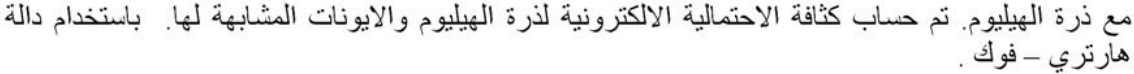

كلمات مفتاحيه : دالة هارتري - فوك , القيمة المتوقعة , كثافة الاحتمالية

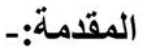

عندما تساوي واحد (شرط العيارية ) و هذا يعني $\int^{\infty} D\left(r_{1}\right) r_{1}^{0} d r_{1}=\int^{\infty} D\left(r_{1}\right) d r_{1}=1 \ldots .(4)$ اللقيم المتوقعة اهمية عندما تحسب لمناطق قطريجة مختلفة حيث بمكن الاستفادة منها في مقارنة احتمالية تواجد الاكترونات في المناطق القريبة من النواة والمناطق البعيدة في كثافة الثحنة الالكترونية لكل غلاف الكتروني وفي، حساب

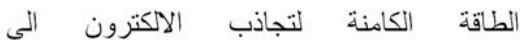
النواة electron-nuclear attraction energy من خلال تحسب من دالتين موجيتين تشير الى كيفية تشابه

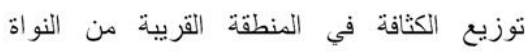
و $\left\langle r_{1}^{+3}\right\rangle$ المنطقة البعيدة في سحابة الثحنة الاككترونية . لتعية
تم دراسة دالة التوزيع القطرية لجسيم واحد

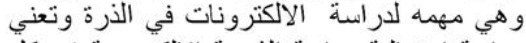

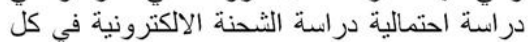
غلاف وقد تم في هذا البحث دراسة التحة هذه ألدالة

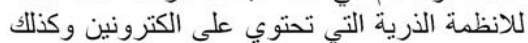

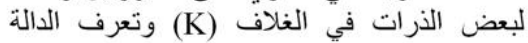
كالاتي : [1] $D\left(r_{l}\right)=\int_{0} \int_{0} r_{l}^{2} \rho\left(r_{l}\right) d \Omega=4 \pi r_{l}^{2} \rho\left(r_{l}\right) \ldots .(1)$

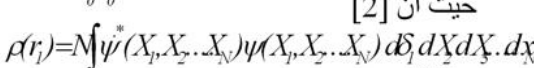

حيث ان الىى مجموعة الاحداثيات الفضائية و والحركة الفئ المغزلية و $)$ عيارية .

ويستفاد من حساب توزيع الكثافة لجسيم واحد

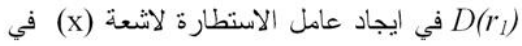
ايجاد القيم المتوقعة لجسيم واحد $-2 \leq n \leq+2$

وكما تم حساب القيمة المتوقعة لجسيم واحد

[3 [ التي يمكن التعبير عنها بالاتي $\left\langle r_{1}^{n}\right\rangle$

$\left\langle r_{1}^{n}\right\rangle=\int_{0}^{\infty} D\left(r_{1}\right) r_{1}^{n} d r_{1}$

*قسم الفيزياء، كلية العلوم للبنات، جامعة بغداد 


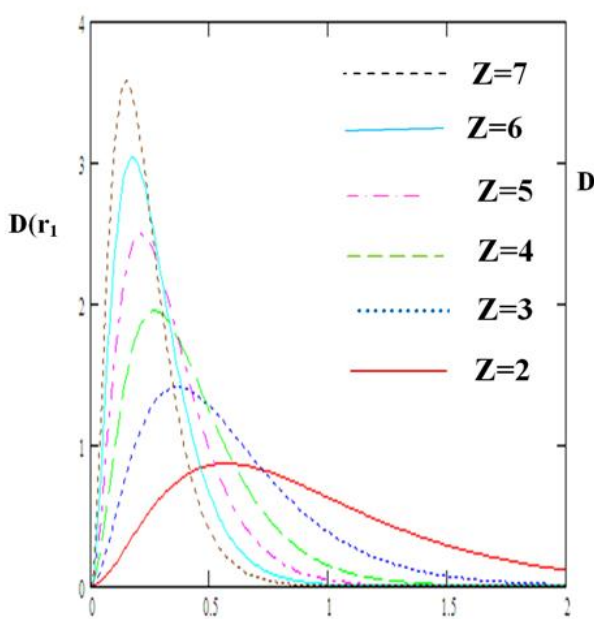

$\mathbf{r}_{1}$

شكل (2) يوضح كثافة الاحتمالية لوجود

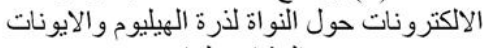

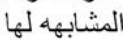

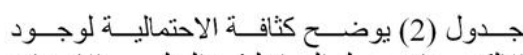
الالكترونـات حول النول النواة لذرة الهيليوم والايونـات

\begin{tabular}{|c|c|c|c|}
\hline Atom or ion & $\mathbf{Z}$ & $\mathbf{r}_{1}$ & $\mathbf{D}\left(\mathbf{r}_{1}\right)_{\max }$ \\
\hline $\mathrm{He}$ & 2 & 0.58 & 0.866 \\
\hline $\mathrm{Li}^{+}$ & 3 & 0.37 & 1.407 \\
\hline $\mathrm{Be}^{+2}$ & 4 & 0.27 & 1.948 \\
\hline $\mathrm{B}^{+3}$ & 5 & 0.23 & 2.471 \\
\hline $\mathrm{C}^{+4}$ & 6 & 0.19 & 3.008 \\
\hline $\mathrm{N}^{+5}$ & 7 & 0.15 & 3.572 \\
\hline
\end{tabular}

جدول (1) يوضح كثافة الاحتمالية لوجود الالكترونات للقترة K حول النواة لبعض الذرات

\begin{tabular}{|c|c|c|c|}
\hline Atom & $\mathbf{Z}$ & $\mathbf{r}_{1}$ & $\mathbf{D}\left(\mathbf{r}_{1}\right)_{\max }$ \\
\hline $\mathrm{He}$ & 2 & 0.58 & 0.866 \\
\hline $\mathrm{Li}$ & 3 & 0.37 & 1.407 \\
\hline $\mathrm{Be}$ & 4 & 0.27 & 1.948 \\
\hline $\mathrm{B}$ & 5 & 0.23 & 2.471 \\
\hline $\mathrm{C}$ & 6 & 0.19 & 3.008 \\
\hline $\mathrm{N}$ & 7 & 0.15 & 3.572 \\
\hline
\end{tabular}




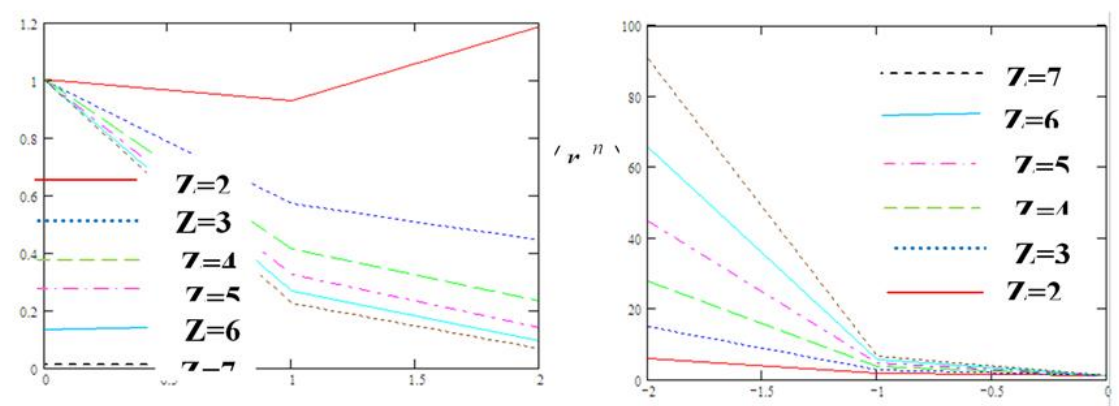

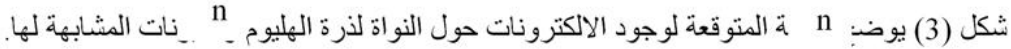

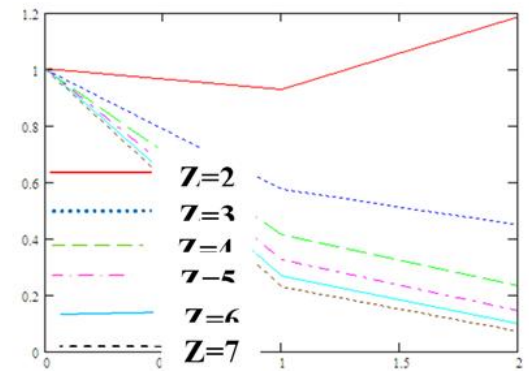

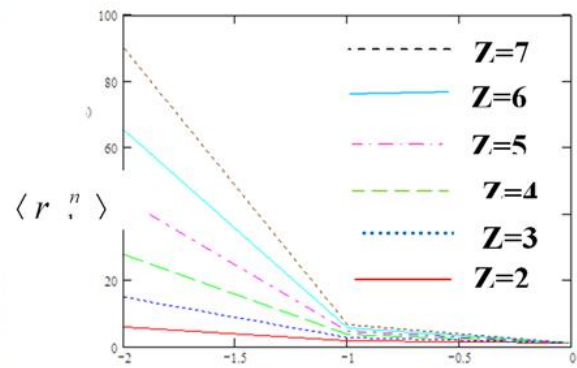

n

شكل (4) يوضح القيمة المتوقعة لوجود الالكترونات للقشرة K حول النواة لبعض الذرات

جدول (3) يوضح القيمة المتوقعة لوجود الالكترونات حول النواة لذرة الهيليوم والانظمة

\begin{tabular}{|c|c|c|c|c|c|c|}
\hline Atom & $\mathbf{Z}$ & $\mathbf{n}=-\mathbf{2}$ & $\mathbf{n = - 1}$ & $\mathbf{n}=\mathbf{0}$ & $\mathbf{n = 1}$ & $\mathbf{n = 2}$ \\
\hline $\mathrm{He}$ & $\mathbf{2}$ & 5.995 & 1.687 & 1.000 & 0.927 & 1.184 \\
\hline $\mathrm{Li}^{+}$ & $\mathbf{3}$ & 14.910 & 2.687 & 1.000 & 0.572 & 0.445 \\
\hline $\mathrm{Li}$ & $\mathbf{3}$ & 14.888 & 2.685 & 1.000 & 0.573 & 0.446 \\
\hline $\mathrm{Be}^{+2}$ & $\mathbf{4}$ & 27.825 & 3.687 & 1.000 & 0.414 & 0.232 \\
\hline $\mathrm{Be}$ & $\mathbf{4}$ & 27.753 & 3.681 & 1.000 & 0.414 & 0.232 \\
\hline $\mathrm{B}^{+3}$ & $\mathbf{5}$ & 44.738 & 4.687 & 1.000 & 0.324 & 0.141 \\
\hline $\mathrm{B}$ & $\mathbf{5}$ & 44.538 & 4.674 & 1.000 & 0.325 & 0.143 \\
\hline $\mathrm{C}^{+4}$ & $\mathbf{6}$ & 65.654 & 5.687 & 1.000 & 0.267 & 0.096 \\
\hline $\mathrm{C}$ & $\mathbf{6}$ & 65.234 & 5.664 & 1.000 & 0.268 & 0.097 \\
\hline $\mathrm{N}^{+5}$ & $\mathbf{7}$ & 90.569 & 6.687 & 1.000 & 0.226 & 0.069 \\
\hline $\mathrm{N}$ & $\mathbf{7}$ & 89.841 & 6.653 & 1.000 & 0.228 & 0.070 \\
\hline
\end{tabular}


القيمة المتوقعة عندما n تأخذ القيم السيالبة

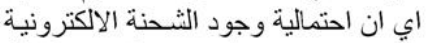

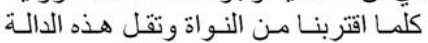

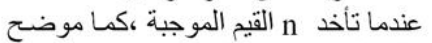

بالجدول ( 3 ) و والثكل (6,4)

2. عند كل عملية تأين تزداد

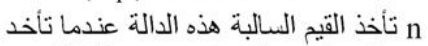

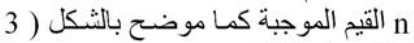

) و الجدول (3 )

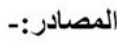

1-Frederick W.King. 1991. Phys. Rev, 44,5 .

2- N.H.March.2003. J. of Chemical physics, 118,15.

3- K .H.Al-Bayati. 2004.J of Um

Salama for Science, 1,2 .

4- - C.F .Bunge, J.A.Barrientos, and

A.V. Bunge 1993, Atomic Data

Nucl.Data Table 53,1

5-- C.C.J.Roothaan , and A.W.Weiss.

1960. Reviews of Modern physics,

32,2 .

6- B. H.Al-assad,K. H.Al-bayati,S.

A.Hasson.2005, J of Um Salama for

Science, 4,3
الحسابات والنتائج:

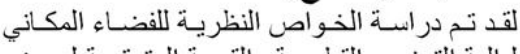

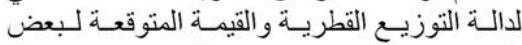

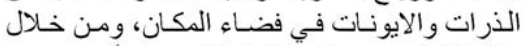
ملاحظة الرسوم والجداول نستنتج مان ما يأتي:-

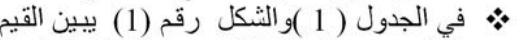

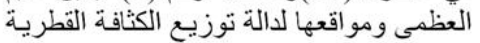

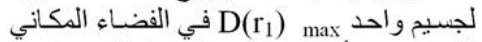
للغلاف K(

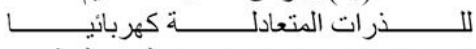

زيـادة القـيم العظمـى (N,C,B,Be,Li,He)

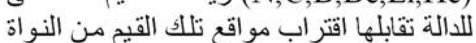

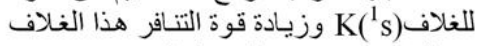

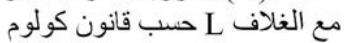

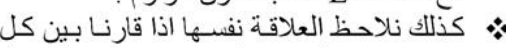

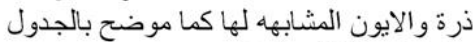

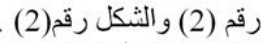

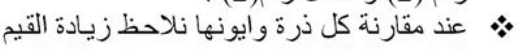

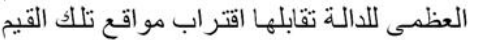

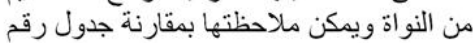

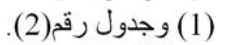

• • من خلال دراسـة القيمة المتوقعة لجسيم واحد الذرة الهليوم والايونات المثابهه

الاستنتاجات

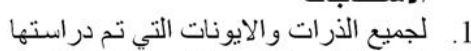
في هذا البحث بزيادة العدد الذري تز تزداد

\title{
Calculate the one-expectation to electronic charge of atomic system contiun two electron
}

\section{Ban.H.Adel* K.H.Al-bayati*}

* physics Dept., College of Science for Women, Baghdad University

\begin{abstract}
:-
The aim of this work is to calculate the one- electron expectation value $\left\langle r_{1}^{n}\right\rangle$ of the electronic charge of atomic system $Z=2,3 \ldots .7$ and we compare with $\mathrm{He}$ atom . the electronic density function $\mathrm{D}\left(\mathrm{r}_{1}\right)$ of $\mathrm{He}$ atom and like ions are evaluated . using Hartree-Fock wave.
\end{abstract}

